\title{
A Novel Nomogram including AJCC Stages Could Better Predict Survival for NSCLC Patients Who Underwent Surgery: A Large Population-Based Study
}

\author{
Xiaoling Shang $\mathbb{D}^{1,2}$ Haining Yu, ${ }^{3}$ Jiamao Lin, ${ }^{4}$ Zhenxiang Li, ${ }^{5}$ Chenglong Zhao, ${ }^{6}$ \\ Jian Sun $\left(\mathbb{1},{ }^{7}\right.$ and Haiyong Wang $\mathbb{B}^{4}$ \\ ${ }^{1}$ Department of Clinical Laboratory, Shandong University, Jinan 250012, China \\ ${ }^{2}$ Department of Clinical Laboratory, Shandong Cancer Hospital and Institute, \\ Shandong First Medical University and Shandong Academy of Medical Sciences, Jinan 250117, China \\ ${ }^{3}$ Personnel Division, Shandong Cancer Hospital and Institute, \\ Shandong First Medical University and Shandong Academy of Medical Sciences, Jinan 250117, China \\ ${ }^{4}$ Department of Internal Medicine-Oncology, Shandong Cancer Hospital and Institute, \\ Shandong First Medical University and Shandong Academy of Medical Sciences, Jinan 250117, China \\ ${ }^{5}$ Department of Radiation Oncology, Shandong Cancer Hospital and Institute, \\ Shandong First Medical University and Shandong Academy of Medical Sciences, Jinan 250117, China \\ ${ }^{6}$ Department of Pathology, Shandong Cancer Hospital and Institute, \\ Shandong First Medical University and Shandong Academy of Medical Sciences, Jinan 250117, China \\ ${ }^{7}$ Department of Thoracic Surgery, Shandong Cancer Hospital and Institute, \\ Shandong First Medical University and Shandong Academy of Medical Sciences, Jinan 250117, China
}

Correspondence should be addressed to Jian Sun; sunjian201911@163.com and Haiyong Wang; wanghaiyong6688@126.com

Received 4 March 2020; Accepted 16 April 2020; Published 20 May 2020

Academic Editor: Dali Zheng

Copyright (C) 2020 Xiaoling Shang et al. This is an open access article distributed under the Creative Commons Attribution License, which permits unrestricted use, distribution, and reproduction in any medium, provided the original work is properly cited.

Objective. In this study, we aimed to establish a novel nomogram model which was better than the current American Joint Committee on Cancer (AJCC) stage to predict survival for non-small-cell lung cancer (NSCLC) patients who underwent surgery. Patients and Methods. 19617 patients with initially diagnosed NSCLC were screened from Surveillance Epidemiology and End Results (SEER) database between 2010 and 2015. These patients were randomly divided into two groups including the training cohort and the validation cohort. The Cox proportional hazard model was used to analyze the influence of different variables on overall survival (OS). Then, using R software version 3.4.3, we constructed a nomogram and a risk classification system combined with some clinical parameters. We visualized the regression equation by nomogram after obtaining the regression coefficient in multivariate analysis. The concordance index (C-index) and calibration curve were used to perform the validation of nomogram. Receiver operating characteristic (ROC) curves were used to evaluate the clinical utility of the nomogram. Results. Univariate and multivariate analyses demonstrated that seven factors including age, sex, stage, histology, surgery, and positive lymph nodes (all, $P<0.001)$ were independent predictors of OS. Among them, stage $(\mathrm{C}$-index $=0.615)$, positive lymph nodes $(\mathrm{C}$-index $=0.574)$, histology $(\mathrm{C}$-index $=0.566)$, age $(\mathrm{C}$-index $=0.563)$, and sex $(\mathrm{C}$-index $=0.562)$ had a relatively strong ability to predict the OS. Based on these factors, we established and validated the predictive model by nomogram. The calibration curves showed good consistency between the actual OS and predicted OS. And the decision curves showed great clinical usefulness of the nomogram. Then, we built a risk classification system and divided NSCLC patients into two groups including high-risk group and low-risk group. The Kaplan-Meier curves revealed that OS in the two groups was accurately differentiated in the training cohort $(P<0.001)$. And then, we validated this result in the validation cohort which also showed that patients in the high-risk group had worse survival than those in the low-risk group. Conclusion. The results proved that the nomogram model had better performance to predict survival for NSCLC patients who underwent surgery than AJCC stage. These tools may be helpful for clinicians to evaluate prognostic indicators of patients undergoing operation. 


\section{Introduction}

NSCLC accounts for about $85 \%$ of all lung cancer, which remains the leading cause of cancer-related death in the world $[1,2]$. In recent years, with the wide application of high-resolution spiral computed tomography (CT) screening technology, the detection rate of early lung cancer has increased significantly [3]. Surgery treatment is the first choice for patients diagnosed with early NSCLC, including stage I, stage II, and partial stage III cases. [4] The current treatment options for NSCLC mainly depend on the eighth edition of the American Joint Committee on Cancer TNM staging. However, patients' survival rate varies greatly at the same stage [5-7]. The 5-year survival rates range from $60 \%$ of stage I to about $30 \%$ of stage IIIA [8,9]. And patients with the same stage showed different rates of survival. It is of great significance in guiding clinical treatment to find independent prognostic factors. Previous studies [5-7] have reported that some factors may significantly promote the survival prediction of patients, such as age, race, sex, stage, and histology.

Nomogram is a convenient tool to predict and quantify risk for patients' prognosis by incorporating and validating some relevant factors. In some other types of tumors, nomograms that calculate numerical probability of clinical events, such as cancer-specific survival (CSS) and OS, have shown more precise prediction than the traditional TNM staging systems. At present, AJCC TNM staging is the main criterion to guide the treatment and prognosis of NSCLC patients. However, the staging could not be good to predict the survival for these patients. Other variables including age, sex, and histology may be significant independent prognostic factors for NSCLC patients. Therefore, the combination of AJCC staging and these variables may be better to predict the outcomes and it would be better in clinical guidance.

Therefore, in the present study, we built and validated the nomogram combined with several clinical variables to predict prognosis for patients with NSCLC who underwent surgery.

\section{Materials and Methods}

2.1. Data Source. The SEER Program (http://www.seer. cancer.gov) consists of 9 Regs Research Data in the United States [10]. Information for patients with stages I-III NSCLC between 2010 and 2015 was extracted from the SEER database. According to the AJCC criteria, we selected a total of 19617 patients diagnosed with NSCLC using the SEER*Stat 8.3.5 software. The inclusion criteria for recruiting patients were as follows: NSCLC patients, only one malignant primary lesion, available clinical information, and active follow-up. The exclusion criteria were patients with benign tumor. In addition, patients containing any missing information on extracted data were all excluded.

2.2. Ethics Statement. Our study was constructed in accordance with the Helsinki Declaration. This study was also approved by the ethics committee of the Shandong Cancer Hospital. This study did not involve any personal information, and therefore, informed patient consent was not required.

2.3. Statistical Analysis. These eligible patients were randomly divided into the training cohort $(70 \%, n=13732)$ and the validation cohort $(30 \%, n=5885)$ to establish and validate the nomogram. The OS was defined as the time from diagnosis to death due to any reason. The data in training cohort were used to develop the prediction model and construct nomogram and risk classification system. Furthermore, the data of the validation cohort were used to make a validation.

Univariate and multivariate analyses were used to determine independent prognostic variables. And then, based on these variables contained in the final model, we built the nomogram and the risk classification system. The C-index was used to determine discrimination ability of the nomogram, and each parameter and ROC curves were used to evaluate the clinical utility of the nomogram. The calibration for 1-, 3-, and 5-year OS was evaluated using a calibration curve by comparing the predicted survival and the observed survival. Furthermore, based on the total score of each patient in the validation cohort, the risk classification system was established and all patients were divided into low-risk and high-risk prognosis groups. The OS was estimated using the Kaplan-Meier method and compared by the log-rank test.

All statistical analyses were made using $\mathrm{R}$ software version 3.4.3 (R Foundation) and Statistical Product Service Solutions (SPSS) 22.0 software package. All statistical $P$ values were 2 -sided, and $P<0.05$ was considered statistically significant.

\section{Results}

3.1. Patients Characteristics. A total of 19617 patients initially diagnosed with NSCLC from the SEER database were included for analysis. All enrolled patients were randomly divided into the training cohort $(13732,70 \%)$ and the validation cohort $(5885,30 \%)$. According to age, all patients were divided into four groups including $<60$ years old ( $n=4203,21.4 \%), 60-69$ years old $(n=7054,36.0 \%), 70-79$ years old $(n=6588,33.6 \%)$, and $>80$ years old $(n=1772$, $33.6 \%)$. In the total cohort, training cohort, and validation cohort, the proportion of patients aged $60-69(36.0 \%, 36.1 \%$ and 35.6, respectively) was the largest. The majority of cases were white $(n=16312,83.2 \%)$. Male and female patients accounted for the same proportion (50\% vs. $50 \%$ ).

According to the AJCC stage, patients of stage T1 were the largest in the total cohort, training cohort, and validation cohort ( $58.8 \%, 58.6 \%$, and 59.4 respectively), followed by the T2 stage (23.3\%, $23.5 \%$, and $22.9 \%$, respectively). And patients with stage T3 was the least in the total cohort, training cohort, and validation cohort $(17.9 \%, 17.9 \%$, and $17.7 \%$, respectively). 12278 (62.6\%) patients had adenocarcinoma 
and $7336(37.4 \%)$ had squamous. 5.6\% of patients underwent complete surgical resection, and $94.4 \%$ of patients underwent partial surgical resection. Of these patients, only $24.5 \%$ patients had positive lymph nodes. Baseline clinicopathological characteristics of all patients in the training cohort and the validation cohort are shown in Table 1.

3.2. Independent Prognostic Factors in Predicting OS. Univariate and multivariate Cox proportional hazards regression models were used to assess each factor's ability in predicting OS. In univariate analysis, we found that age $(P<0.001)$, race $(P<0.001), \quad$ sex $\quad(P=0.03)$, stage $(P<0.001)$, histology $(P<0.001)$, surgery $(P<0.001)$, and positive lymph nodes $(P<0.001)$ were associated with OS in patients with stages I-III NSCLC. Among them, stage (Cindex $=0.615)$, positive lymph nodes $(\mathrm{C}$-index $=0.574)$, histology $(\mathrm{C}$-index $=0.566)$, age $(\mathrm{C}$-index $=0.563)$, and sex $(\mathrm{C}$-index $=0.562)$ had superior discrimination power in predicting OS compared with other variables. Multivariate analysis further analyzed the factors of a $P<0.05$ in univariate analysis. In the multivariate analysis, we found that age $(P<0.001)$, other races $(P<0.001)$, sex $(P<0.001)$, stage $(P<0.001)$, histology $(P<0.001)$, surgery $(P<0.001)$, and positive lymph nodes $(P<0.001)$ were independent prognostic factors and were incorporated into the predictive model. Univariate and multivariate analyses of each factor's ability in predicting OS are shown in Table 2.

\subsection{Building and Validating the Predictive Nomogram.} We built a novel nomogram that included the significant and independent prognostic factors (Figure 1). Each factor had a score on the point scale. We can draw a straight line to determine the estimated probability of prognosis at each time point by adding up the total score and locating it on the total point scale. And then, the validation cohort was used to verify the novel nomogram. In the validation cohort, we compared the OS rate predicted by the nomogram with observed 1-, 3-, and 5-year OS rates.

In a well-calibrated model, the prediction will fall on a 45-degree diagonal line. From Figure 2, the calibration curves revealed good consistency between the actual observation and the nomogram prediction for 1-, 3-, and 5year survival rates. Figure 2(a) shows good consistency between the actual 1-year overall survival and predicted 1year overall survival. And the ROC curve revealed that the area under the curve (AUC) is 0.701 . Figure 2(b) shows good consistency between the actual 3-year overall survival and predicted 3-year overall survival. And the ROC curve revealed that the AUC is 0.687. Figure 2(c) shows good consistency between the actual 5-year overall survival and predicted 5-year overall survival. And the ROC curve revealed that the AUC is 0.669 .

In addition, decision curves exhibited great positive net benefits in the predictive model among almost all of the threshold probabilities at different time points, indicating the favorable potential clinical effect of the predictive model (Figures 3(a) and 3(b)).
3.4. Risk Classification System. According to the total scores of every patient, we also developed a risk classification system in the training cohort generated by nomogram. All patients in the training cohort and validation cohort were divided into the high-risk and low-risk groups. The Kaplan-Meier curve was used to draw the OS curves for the high-risk group and low-risk group in the training cohort and validation cohort. In the training cohort, the Kaplan-Meier curves revealed that patients' survival in the low-risk group was better than that in the high-risk group $(P<0.001)$ (Figure 4(a)). Then, we validated it in the validation cohort. Similarly, patients in the low-risk group had better survival than those in the high-risk group $(P<0.001)$ (Figure 4(b)).

\section{Discussion}

In this study, we established and developed a nomogram and a risk classification to predict the OS of patients with stages I-III NSCLC after surgery using the data originated from SEER database. A total of 19167 patients were included, and seven significant prognosis factors including age, race, sex, stage, histology, surgery, and positive nodes were identified. And these predictive factors could be easily obtained from clinical practice. Then, we established the validation of model and used different statistical methods to demonstrate its great performance.

Over time, the prospects for lung cancer patients and treatment have changed. Lung lobectomy is often considered the best treatment option for stages I, II, and partial III NSCLC patients $[7,8,11]$. Recurrence and metastasis have become important factors affecting the 5 -year survival rate of patients with lung cancer after operation. So, it is very important to predict factors of survival after surgery in NSCLC patients. Furthermore, NSCLC has significant heterogeneity in individual survival, and it is inaccurate to use the TNM staging system to predict survival. Although several prognostic models have been reported previously $[6,12]$, a relevant nomogram was rarely developed to predict prognostic variables for patients NSCLC after surgery.

Some research studies [13-18] reported that a nomogram could predict the prognosis of NSCLC patients. However, most studies focused on patients with early or advanced NSCLC. Nonetheless, both research studies had a small sample size which may inhibit their generalization.

Liang et al. [19] showed that the C-index for the established nomogram to predict OS was 0.71 in the primary cohort and 0.67 in the IASLC cohort. Sun et al. [13] showed that the C-index of the nomogram was 0.638 which exhibited a sufficient level of discrimination. However, in our study, the C-index of the nomogram is higher than that of other previous models. In addition to a nomogram, we also developed a risk classification system and the risk classification divided the whole NSCLC patients into two distinct prognostic groups which could supplement the nomogram in our study.

In univariable and subsequent multivariable analysis, we identified age, race, sex, stage, histology, surgery types, and positive lymph nodes as independent prognostic factors. 
TABLE 1: Baseline clinicopathological characteristics of all patients and those in the training and validation cohorts.

\begin{tabular}{|c|c|c|c|c|}
\hline Variables & All cohort $(n=19617)$ & Training cohort $(n=13732)$ & Validation cohort $(n=5885)$ & $P$ \\
\hline $\begin{array}{l}\text { Age } \\
<60 \\
60-69 \\
70-79 \\
>80\end{array}$ & $\begin{array}{c}4203(21.4) \\
7054(36.0) \\
6588(33.6) \\
1772(9.0)\end{array}$ & $\begin{array}{l}4958(36.1) \\
4619(33.6) \\
1254(9.1)\end{array}$ & $\begin{array}{c}1302(22.1) \\
2096(35.6) \\
1969(33.5) \\
518(8.8)\end{array}$ & 0.026 \\
\hline $\begin{array}{l}\text { Race } \\
\text { White } \\
\text { Black } \\
\text { Others }\end{array}$ & $\begin{array}{c}16312(83.2) \\
1814(9.2) \\
1491(7.6) \\
\end{array}$ & $\begin{array}{r}11445(83.3) \\
1262(9.2) \\
1025(7.5) \\
\end{array}$ & $\begin{array}{c}4867(82.7) \\
552(9.4) \\
466(7.9) \\
\end{array}$ & 0.019 \\
\hline $\begin{array}{l}\text { Sex } \\
\text { Male } \\
\text { Female } \\
\end{array}$ & $\begin{array}{l}9807(50.0) \\
9810(50.0) \\
\end{array}$ & $\begin{array}{l}6839(49.8) \\
6893(50.2) \\
\end{array}$ & $\begin{array}{l}2968(50.4) \\
2917(49.6) \\
\end{array}$ & 0.013 \\
\hline $\begin{array}{l}\text { Stage } \\
\text { I } \\
\text { II } \\
\text { III } \\
\end{array}$ & $\begin{array}{c}11543(58.8) \\
4572(23.3) \\
3502(17.9) \\
\end{array}$ & $\begin{array}{l}8047(58.6) \\
3226(23.5) \\
2459(17.9) \\
\end{array}$ & $\begin{array}{l}3496(59.4) \\
1346(22.9) \\
1043(17.7) \\
\end{array}$ & 0.017 \\
\hline $\begin{array}{l}\text { Histology } \\
\text { Adenocarcinoma } \\
\text { Squamous }\end{array}$ & $\begin{array}{c}12278(62.6) \\
7336(37.4) \\
\end{array}$ & $\begin{array}{l}8579(62.5) \\
5153(37.5) \\
\end{array}$ & $\begin{array}{l}3702(62.9) \\
2183(37.1) \\
\end{array}$ & 0.009 \\
\hline $\begin{array}{l}\text { Surgery } \\
\text { Complete resection } \\
\text { Partial resection } \\
\text { Positive nodes } \\
\text { Yes } \\
\text { No } \\
\end{array}$ & $\begin{array}{c}1092(5.6) \\
18525(94.4) \\
4812(24.5) \\
14805(75.5) \\
\end{array}$ & $\begin{array}{c}778(5.7) \\
12954(94.3)\end{array}$ & $\begin{aligned} & 314(5.3) \\
& 5571(94.7) \\
& 1452(24.7) \\
& 4433(75.3) \\
&\end{aligned}$ & $\begin{array}{l}0.014 \\
0.005\end{array}$ \\
\hline
\end{tabular}

TABLE 2: Univariate and multivariate analyses of each factor's ability in predicting OS.

\begin{tabular}{|c|c|c|c|c|c|c|c|}
\hline \multirow[b]{2}{*}{ Variable } & \multicolumn{4}{|c|}{ Univariate analyses } & \multicolumn{3}{|c|}{ Multivariate analyses } \\
\hline & HR & $95 \% \mathrm{CI}$ & $P$ & C-index & HR & $95 \% \mathrm{CI}$ & $P$ \\
\hline Age & & & & 0.563 & & & \\
\hline$<60$ & Reference & & & & Reference & & \\
\hline $60-69$ & 1.110 & $1.010-1.220$ & 0.038 & & 1.174 & $1.065-1.294$ & 0.001 \\
\hline $70-79$ & 1.430 & $1.300-1.570$ & $<0.001$ & & 1.604 & $1.455-1.768$ & $<0.001$ \\
\hline$>80$ & 2.00 & $1.780-2.260$ & $<0.001$ & & 2.367 & $2.095-2.674$ & $<0.001$ \\
\hline Race & & & & 0.516 & & & \\
\hline White & Reference & & & & Reference & & \\
\hline Black & 0.913 & $0.813-1.025$ & 0.120 & & 1.022 & $0.909-1.148$ & 0.717 \\
\hline Others & 0.748 & $0.649-0.863$ & $<0.001$ & & 0.777 & $0.673-0.897$ & $<0.001$ \\
\hline Sex & & & & 0.562 & & & \\
\hline Male & Reference & & & & Reference & & \\
\hline Female & 0.649 & $0.607-0.694$ & 0.030 & $<0.001$ & 0.714 & $0.667-0.764$ & $<0.001$ \\
\hline Stage & & & & 0.615 & & & \\
\hline I & Reference & & & & Reference & & \\
\hline II & 2.100 & $1.940-2.270$ & $<0.001$ & & 1.832 & $1.672-2.006$ & $<0.001$ \\
\hline III & 2.610 & $2.410-2.830$ & $<0.001$ & & 2.287 & $2.047-2.554$ & $<0.001$ \\
\hline Histology & & & & 0.566 & & & \\
\hline Adenocarcinoma & Reference & & & & Reference & & \\
\hline Squamous & 1.570 & $1.470-1.6770$ & $<0.001$ & & 1.325 & $1.237-1.420$ & $<0.001$ \\
\hline Surgery & & & & 0.528 & & & \\
\hline Complete resection & Reference & & & & Reference & & \\
\hline Partial resection & 1.990 & $1.780-2.230$ & $<0.001$ & & 1.297 & $1.150-1.462$ & $<0.001$ \\
\hline Positive nodes & & & $<0.001$ & 0.574 & & & \\
\hline Yes & Reference & & & & Reference & & \\
\hline No & 2.030 & $1.900-2.170$ & & & 1.183 & $1.077-1.299$ & $<0.001$ \\
\hline
\end{tabular}




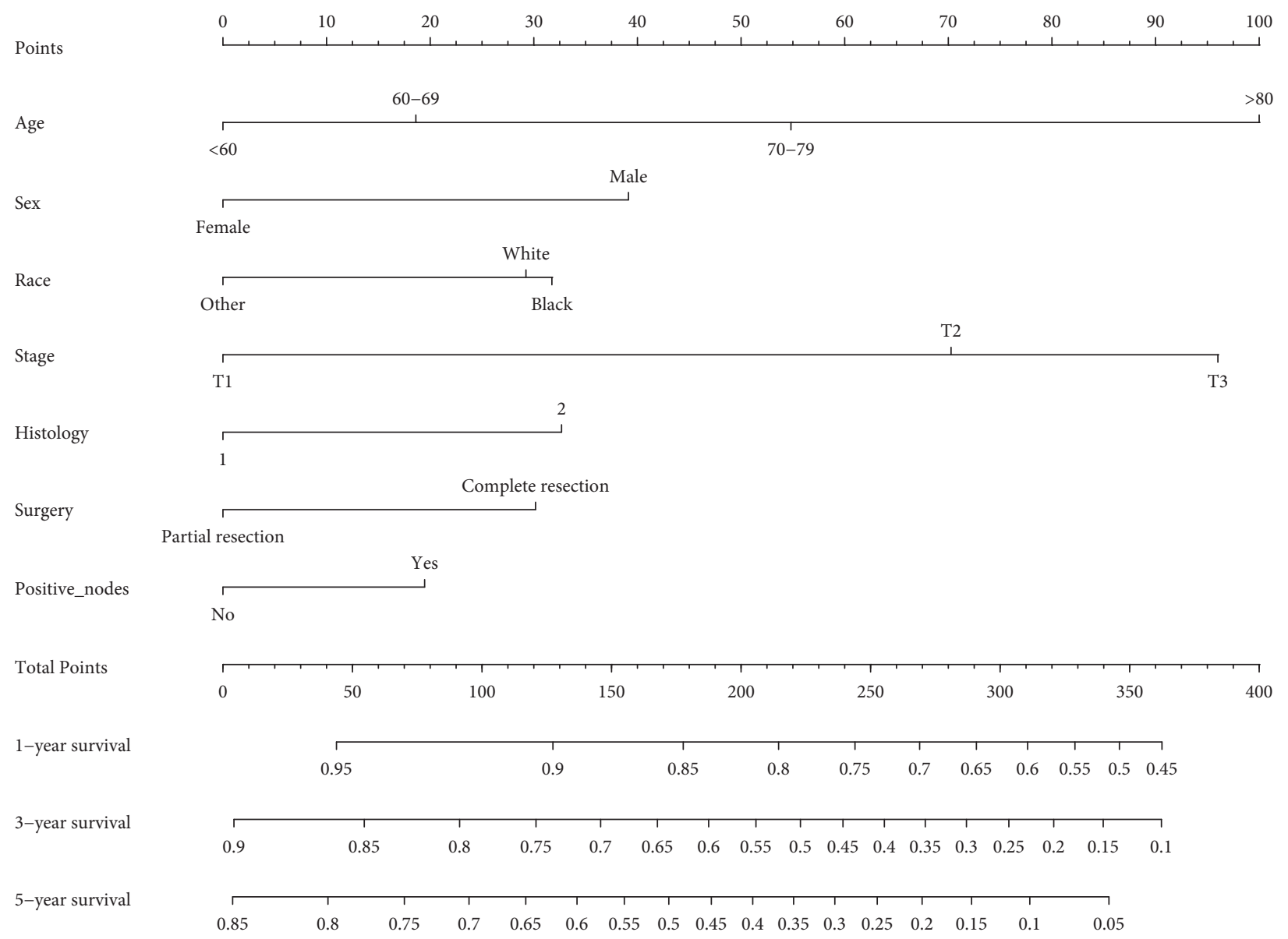

FIGURe 1: A nomogram for prediction of 1-, 3-, and 5-year OS rates of stages I-III NSCLC patients after surgery.
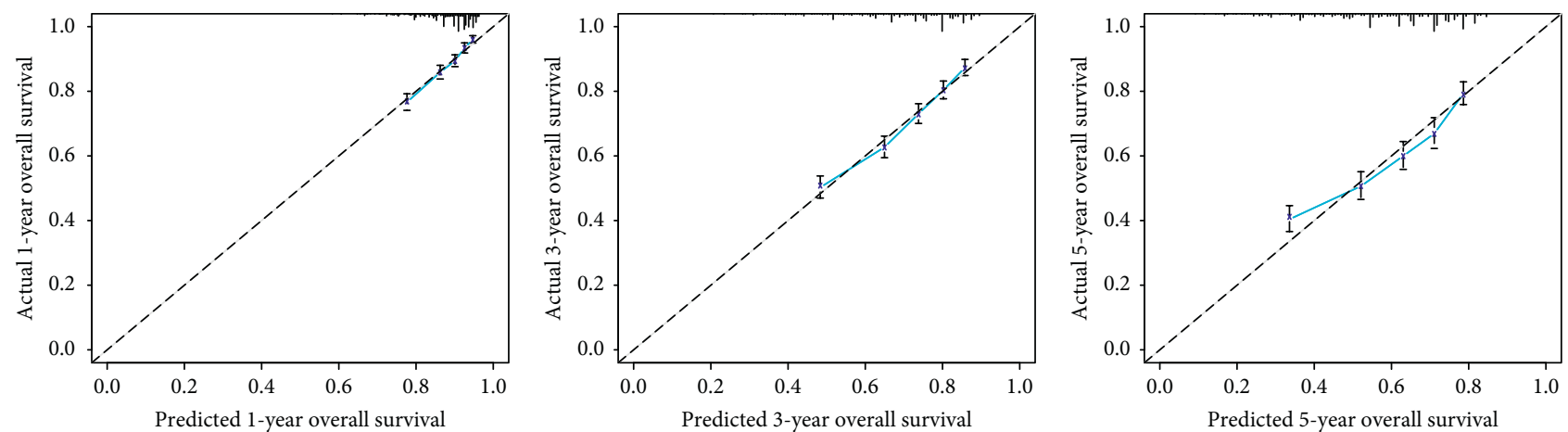

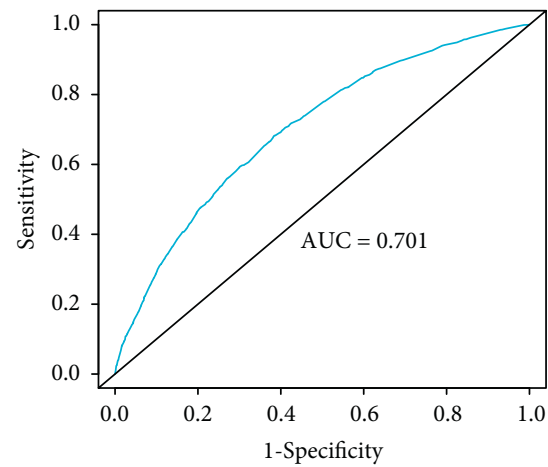

(a)

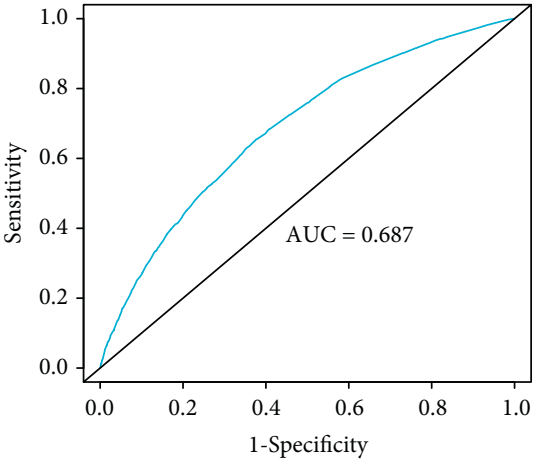

(b)

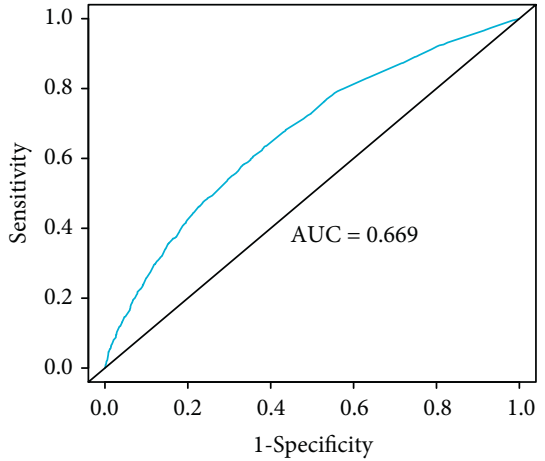

(c)

FIGURE 2: Calibration curves of the nomogram predicting 1-year, 3-year, and 5-year OS rates of stages I-III NSCLC patients after surgery. On the calibration plot, the $x$-axis is nomogram-predicted probability of over survival. The $y$-axis is the actual over survival. 


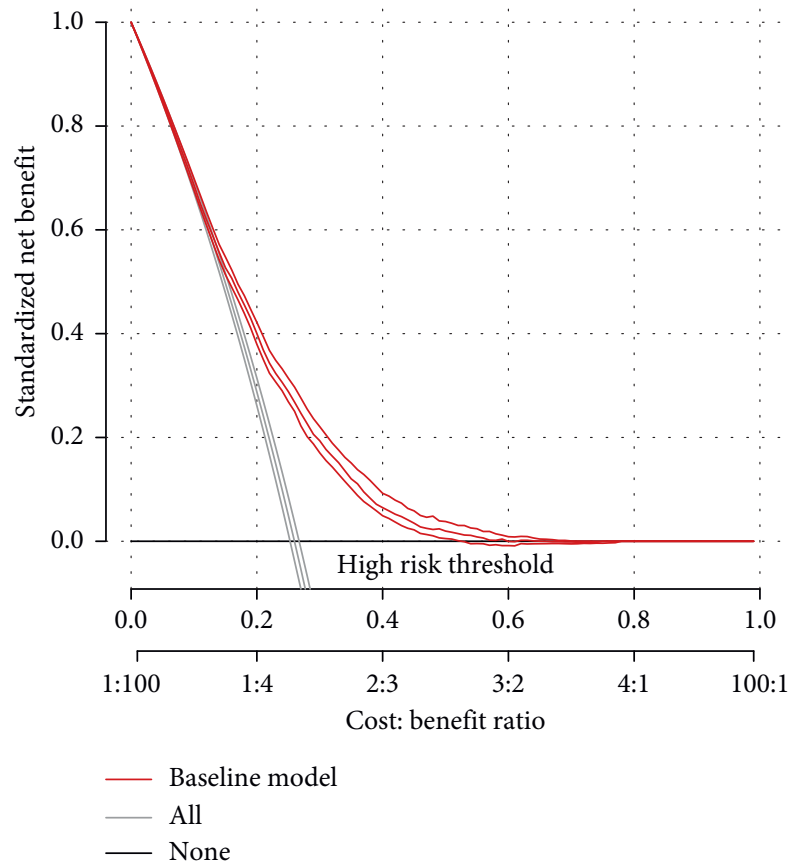

(a)

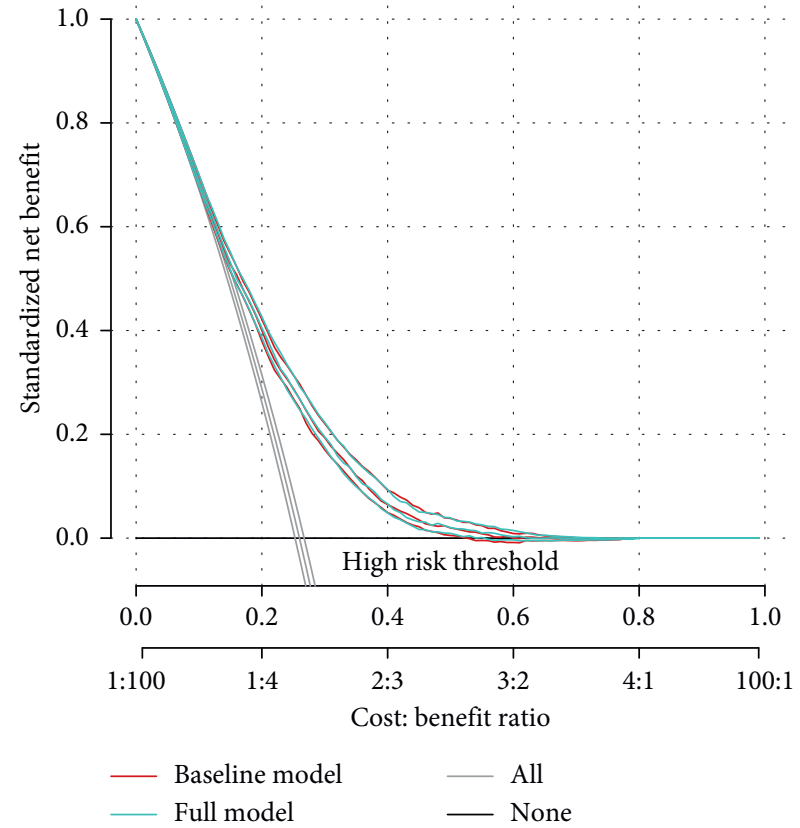

(b)

FIGURE 3: Decision curves of the nomogram predicting OS. The $x$-axis represents the threshold probabilities, and the $y$-axis measures the net benefit calculated by adding the true positives and subtracting the false positives.

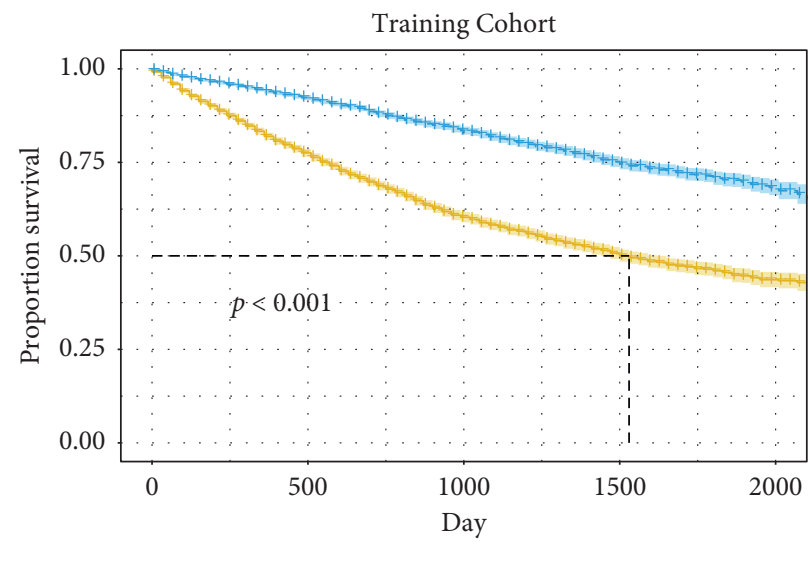

High risk

-+- Low risk

(a)

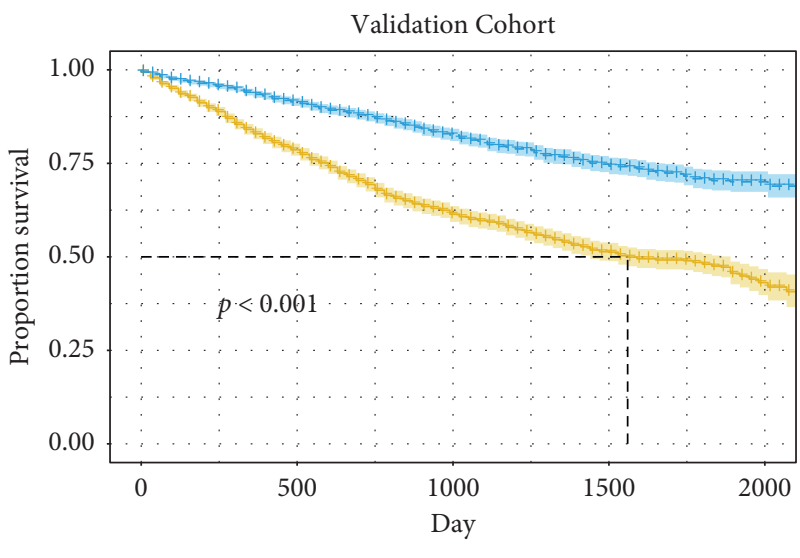

High risk

-+- Low risk

(b)

FIgURE 4: Kaplan-Meier curves of OS for patients in the low- and high-risk groups. (a) Kaplan-Meier curves of OS for patients in the lowand high-risk groups in the training cohort. (b) Kaplan-Meier curves of OS for patients in the low-and high-risk groups in the validation cohort.

These findings are consistent with previous reports on risk factors for non-small-cell lung cancer $[7,8,20]$. It is necessary to validate the nomogram and avoid excessive fitting of the model and determine the extensibility [11]. Notably, according to our nomogram, stage is the most powerful predictor of OS, and C-index $(\mathrm{C}$-index $=0.615)$ was the highest among all predictors. One of the possible reasons is that TNM staging is the current important tool to make decision about the stage-specific therapeutic strategy and assess the prognostic survival [21]. However, in the present study, we did not divide these stages into specific $\mathrm{T}$ and $\mathrm{N}$ category, which were reported as the significant and independent factors in other research studies. We need future studies to assess each factor of stage which may impact on survival for patients with resected NSCLC.

In addition, positive lymph node was another important predictor for OS and the C-index was 0.574. Several research studies $[22,23]$ reported the relationship between 
positive lymph nodes and survival. The reason may be that with more positive lymph nodes being cleared out, potential metastatic lymph nodes will be removed. For patients with resected NSCLC, the number of positive lymph nodes was also demonstrated as an important prognostic factor $[24,25]$. And in many other cancers, positive lymph node is an important factor affecting survival [26-28]. Moreover, complete sampling of lymph nodes results in precise staging and, therefore, appropriate adjuvant treatments for patients.

In this study, we defined 1-, 3-, and 5-year survival rates as our endpoints. Calibration curves showed good agreement between nomogram prediction and actual observation. The nomogram performed well by AUC at every measured time point, which revealed that the nomogram had good performance to predict 1-, 3-, and 5-year OS rates for patients with resected NSCLC. Kaplan-Meier curves showed that OS in the different groups was accurately differentiated by the risk classification system in the training cohort and validation cohort, both of $P<0.05$.

Although surgery is the first choice treatment for patients with stages I, II, and partial III NSCLC [29, 30], postoperative adjuvant treatment could decrease the risk of disease recurrence and improve outcome [30-32]. It should be noted that postoperative adjuvant therapies including chemotherapy, radiotherapy, target treatment, and any other adjuvant therapies were not selected as candidate factors because they were only recommended for a proportion of patients with potentially high risk of locoregional recurrence.

In addition, patients with $\mathrm{N} 2$ disease were a heterogeneous group [33]. Operation may have some limitations for these patients, and the treatment should be individualized [34]. Mao et al. [35] showed that the C-index of the nomogram was 0.673 in the training cohort and 0.664 in the validation cohort. In our study, we did not specify the proportion of these patients with $\mathrm{N} 2$ disease who were treated with surgery from SEER database. The future studies are necessary to validate this result.

However, there are several limitations in our study. First, this was a retrospective study from the SEER database which could not represent the global population. Second, some other factors affecting survival, including smoking history, tumor location, and resection type, were not included in the present study. These data also may have an impact on clinical prognosis. Third, due to the limitations of the SEER database, the details of specific adjuvant therapy, such as chemotherapy and radiochemotherapy which may have some effect on survival for these patients, could not be obtained. Finally, although we use a large cohort to establish the nomogram and risk classification and validated in validation cohort, further validation of the predictive model is still essential.

\section{Conclusion}

We established a nomogram and a corresponding risk classification system predicting survival for NSCLC patients who underwent surgery. The results proved that the model had better performance to predict survival for NSCLC patients who underwent surgery than AJCC stage. Although future validation is necessary, these tools may be helpful for clinicians to evaluate prognostic indicators of patients undergoing operation.

\section{Abbreviations \\ NSCLC: Non-small-cell lung cancer \\ SEER: Surveillance epidemiology and end results \\ OS: Overall survival \\ CT: $\quad$ Computed tomography \\ HR: Hazard ratio \\ CI: $\quad$ Confidence interval \\ AJCC: American Joint Committee on Cancer \\ C-index: Concordance index \\ ROC: Receiver operating characteristic \\ AUC: Area under the curve.}

\section{Data Availability}

The datasets used and analyzed during the current study are available from the corresponding author upon reasonable request.

\section{Conflicts of Interest}

All the authors have no conflicts of interest to declare.

\section{Authors' Contributions}

$\mathrm{X}-\mathrm{L} \mathrm{S}$ was in charge of analysis and wrote the article.H-N Y, $\mathrm{Z}-\mathrm{X} \mathrm{L}$, and J-M L helped with acquisition and analysis of the data. C-L Z helped in editing language. J S and H-Y W, the corresponding authors, were in charge of guidance of the design and analysis the whole research. H-Y W and X-L S were major contributors to the manuscript. All authors read and approved the final manuscript.

\section{Acknowledgments}

This study was supported jointly by Special Funds for Taishan Scholars Project (Grant no. Tsqn201812149) and Academic Promotion Program of Shandong First Medical University (2019RC004).

\section{References}

[1] A. Jemal, R. Siegel, J. Xu, and E. Ward, "Cancer statistics, 2010," CA: A Cancer Journal for Clinicians, vol. 60, no. 5, pp. 277-300, 2010.

[2] L. A. Torre, R. L. Siegel, and A. Jemal, "Lung cancer statistics," Lung Cancer and Personalized Medicine, vol. 893, pp. 1-19, 2016.

[3] P. Goldstraw, J. Crowley, K. Chansky et al., "The IASLC Lung Cancer Staging Project: proposals for the revision of the TNM stage groupings in the forthcoming (seventh) edition of the TNM Classification of malignant tumours," Journal of Thoracic Oncology, vol. 2, no. 8, pp. 706-714, 2007.

[4] T. Kawaguchi, M. Takada, A. Kubo et al., "Performance status and smoking status are independent favorable prognostic 
factors for survival in non-small cell lung cancer: a comprehensive analysis of 26,957 patients with NSCLC," Journal of Thoracic Oncology, vol. 5, no. 5, pp. 620-630, 2010.

[5] K. Chansky, J.-P. Sculier, J. J. Crowley, D. Giroux, J. Van Meerbeeck, and P. Goldstraw, "The International Association for the Study of Lung Cancer Staging Project: prognostic factors and pathologic TNM stage in surgically managed non-small cell lung cancer," Journal of Thoracic Oncology, vol. 4, no. 7, pp. 792-801, 2009.

[6] J.-P. Sculier, K. Chansky, J. J. Crowley, J. Van Meerbeeck, and P. Goldstraw, "The impact of additional prognostic factors on survival and their relationship with the anatomical extent of disease expressed by the 6th edition of the TNM classification of malignant tumors and the proposals for the 7th edition," Journal of Thoracic Oncology, vol. 3, no. 5, pp. 457-466, 2008.

[7] N. Sawabata, H. Asamura, T. Goya et al., "Japanese Lung Cancer Registry Study: first prospective enrollment of a large number of surgical and nonsurgical cases in 2002," Journal of Thoracic Oncology, vol. 5, no. 9, pp. 1369-1375, 2010.

[8] H. Asamura, T. Goya, Y. Koshiishi et al., "A Japanese Lung Cancer Registry study: prognosis of 13,010 resected lung cancers," Journal of Thoracic Oncology, vol. 3, no. 1, pp. 46-52, 2008.

[9] M. Kates, X. Perez, J. Gribetz, S. J. Swanson, T. McGinn, and J. P. Wisnivesky, "Validation of a model to predict perioperative mortality from lung cancer resection in the elderly," American Journal of Respiratory and Critical Care Medicine, vol. 179, no. 5, Article ID 390e395, 2009.

[10] National Cancer Institute, "Surveillance, epidemiology and end results (seer) program," SEER *Stat Database: Incidence SEER 9 Regs Research Data, Nov 2018 Sub (1975-2016) $<$ Katrina/Rita Population Adjustment>-linked to Country Attributes-Total U.S.,1969-2017 Countries, National Cancer Institute, Bethesda, MY, USA, 2019, http://www.seer.cancer. govDCCPS, Surveillance Research Program.

[11] T. Goya, H. Asamura, H. Yoshimura et al., "Prognosis of 6644 resected non-small cell lung cancers in Japan: a Japanese lung cancer registry study," Lung Cancer, vol. 50, no. 2, pp. 227234, 2005

[12] Ö. Birim, A. P. Kappetein, M. Waleboer et al., "Long-term survival after non-small cell lung cancer surgery: development and validation of a prognostic model with a preoperative and postoperative mode," The Journal of Thoracic and Cardiovascular Surgery, vol. 132, no. 3, pp. 491-498, 2006.

[13] F. Sun, K. Ma, X. Yang et al., "A nomogram to predict prognosis after surgery in early stage non-small cell lung cancer in elderly patients," International Journal of Surgery, vol. 42, pp. 11-16, 2017.

[14] T. Wang, R. Lu, S. Lai et al., "Development and validation of a nomogram prognostic model for patients with advanced nonsmall-cell lung cancer," Cancer Informatics, vol. 18, Article ID $1176935119837547,2019$.

[15] A. Botticelli, M. Salati, F. R. Di Pietro et al., "A nomogram to predict survival in non-small cell lung cancer patients treated with nivolumab," Journal of Translational Medicine, vol. 17, no. 1, p. 99, 2019.

[16] Y. Zeng, J. Y. Chi-Fu, N. Mayne, et al.,AME Thoracic Surgery Collaborative Group, "A nomogram for predicting cancerspecific survival of TNM 8th edition stage I non-small-cell lung cancer," Annals of Surgical Oncology, vol. 26, no. 7, pp. 2053-2062, 2019.

[17] H. Yang, X. Li, J. Shi et al., "A nomogram to predict prognosis in patients undergoing sublobar resection for stage IA non- small-cell lung cancer," Cancer Management and Research, vol. 10, pp. 6611-6626, 2018.

[18] J. Deng, Z. Ren, J. Wen et al., "Construction of a nomogram predicting the overall survival of patients with distantly metastatic non-small-cell lung cancer," Cancer Management and Research, vol. 10, pp. 6143-6156, 2018.

[19] W. Liang, L. Zhang, G. Jiang et al., "Development and validation of a nomogram for predicting survival in patients with resected non-small-cell lung cancer," Journal of Clinical Oncology, vol. 33, no. 8, pp. 861-869, 2015.

[20] P. M. Heerdt and B. J. Park, "The emerging role of minimally invasive surgical techniques for the treatment of lung malignancy in the elderly," Anesthesiology Clinics, vol. 26, no. 2, Article ID 315e324, 2008.

[21] P. Goldstraw, K. Chansky, J. Crowley et al., "The IASLC lung cancer staging project: proposals for revision of the TNM stage groupings in the forthcoming (eighth) edition of the TNM classification for lung cancer," Journal of Thoracic Oncology: Official Publication of the International Association for the Study of Lung Cancer, vol. 11, no. 1, pp. 39-51, 2016.

[22] A. Gajra, N. Newman, G. P. Gamble, L. J. Kohman, and S. L. Graziano, "Effect of number of lymph nodes sampled on outcome in patients with stage I non-small-cell lung cancer," Journal of Clinical Oncology, vol. 21, no. 6, pp. 1029-1034, 2003.

[23] R. U. Osarogiagbon, O. Ogbata, and X. Yu, "Number of lymph nodes associated with maximal reduction of long-term mortality risk in pathologic node-negative non-small cell lung cancer," The Annals of Thoracic Surgery, vol. 97, no. 2, pp. 385-393, 2014.

[24] T. Fukui, S. Mori, K. Yokoi, and T. Mitsudomi, "Significance of the number of positive lymph nodes in resected non-small cell lung cancer," Journal of Thoracic Oncology, vol. 1, no. 2, pp. 120-125, 2006.

[25] J. G. Lee, C. Y. Lee, I. K. Park et al., "Number of metastatic lymph nodes in resected non-small cell lung cancer predicts patient survival," The Annals of Thoracic Surgery, vol. 85, no. 1, pp. 211-215, 2008.

[26] R. Vather, T. Sammour, A. Kahokehr, A. B. Connolly, and A. G. Hill, "Lymph node evaluation and long-term survival in stage II and stage III colon cancer: a national study," Annals of Surgical Oncology, vol. 16, no. 3, pp. 585-593, 2009.

[27] S. S. Groth, B. A. Virnig, B. A. Whitson et al., "Determination of the minimum number of lymph nodes to examine to maximize survival in patients with esophageal carcinoma: data from the surveillance epidemiology and end results database," The Journal of Thoracic and Cardiovascular Surgery, vol. 139, no. 3, pp. 612-620, 2010.

[28] M. May, E. Herrmann, C. Bolenz et al., "Association between the number of dissected lymph nodes during pelvic lymphadenectomy and cancer-specific survival in patients with lymph node-negative urothelial carcinoma of the bladder undergoing radical cystectomy," Annals of Surgical Oncology, vol. 18, no. 7, pp. 2018-2025, 2011.

[29] P. E. Van Schil, B. Balduyck, M. De Waele, J. M. Hendriks, M. Hertoghs, and P. Lauwers, "Surgical treatment of earlystage non-small-cell lung cancer," European Journal of Cancer Supplements, vol. 11, no. 2, pp. 110-122, 2013.

[30] J. D. Bradley, R. Paulus, M. V. Graham et al., "Phase II trial of postoperative adjuvant paclitaxel/carboplatin and thoracic radiotherapy in resected stage II and IIIA non-small-cell lung cancer: promising long-term results of the radiation therapy oncology group-RTOG 9705," Journal of Clinical Oncology, vol. 23, no. 15, pp. 3480-3487, 2005. 
[31] Z. Hui, H. Dai, J. Liang et al., "Selection of proper candidates with resected pathological stage IIIA-N2 non-small cell lung cancer for postoperative radiotherapy," Thoracic Cancer, vol. 6, no. 3, pp. 346-353, 2015.

[32] D. R. Gomez and R. Komaki, "Postoperative radiation therapy for non-small cell lung cancer and thymic malignancies," Cancers, vol. 4, no. 1, pp. 307-322, 2012.

[33] F. Andre, D. Grunenwald, J.-P. Pignon et al., "Survival of patients with resected N2 non-small-cell lung cancer: evidence for a subclassification and implications," Journal of Clinical Oncology, vol. 18, no. 16, pp. 2981-2989, 2000.

[34] M. Reif, M. A. Socinski, and M. P. Rivera, "Evidence-based medicine in the treatment of non-small cell lung cancer," Clinics in Chest Medicine, vol. 21, no. 1, pp. 107-120, 2000.

[35] Q. Mao, W. Xia, G. Dong et al., "A nomogram to predict the survival of stage IIIA-N2 non-small cell lung cancer after surgery," The Journal of Thoracic and Cardiovascular Surgery, vol. 155, no. 4, pp. 1784-1792, 2018. 